\title{
Efectos del framing en diseños de realismo experimental. Consumo de encuadres y compromiso político en la campaña electoral mexicana de 2018
}

\section{Framing effects in experimental realism designs: frame consumption and political engagement in the 2018 Mexican election campaign}

\author{
Carlos Muñiz; Martín Echeverría
}

Cómo citar este artículo:

Muñiz, Carlos; Echeverría, Martín (2020). "Efectos del framing en diseños de realismo experimental. Consumo de encuadres y compromiso político en la campaña electoral mexicana de 2018". Profesional de la información, v. 29, n. 6, e290613.

https://doi.org/10.3145/epi.2020.nov.13

Artículo recibido el 21-01-2020 Aceptación definitiva: 12-03-2020

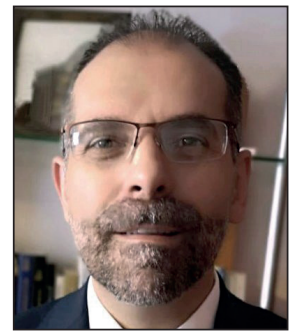

Carlos Muñiz $\square$ https://orcid.org/0000-0002-9021-8198

Universidad Autónoma de Nuevo León Facultad de Ciencias Políticas y Relaciones Internacionales

Campus Mederos, Ave. Praga y Trieste, $\mathrm{s} / \mathrm{n}$

Colonia Residencial Las Torres

64930 Monterrey (Nuevo León), México

carlos.munizm@uanl.mx

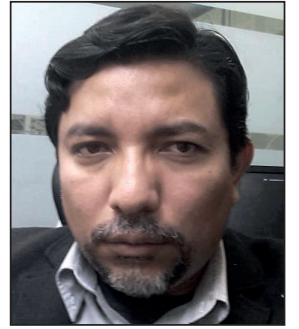

Martín Echeverría

https://orcid.org/0000-0001-6071-8725

Benemérita Universidad Autónoma de Puebla Instituto de Ciencias de Gobierno y Desarrollo Estratégico

Av. Cúmulo de Virgo, s/n.

Complejo Cultural Universitario

72810 Puebla (Puebla), México

echevemartin@yahoo.com.mx

\section{Resumen}

La bibliografía internacional ha permitido determinar la influencia de los medios de comunicación en la generación de actitudes y comportamientos políticos de los ciudadanos, a partir de su cobertura de la política y en particular del framing de las noticias durante las campañas, de tipo asunto político y juego estratégico. Aunque habitualmente estos estudios se han elaborado desde diseños experimentales clásicos, recientemente se ha planteado la necesidad de realizar estudios de mayor validez externa y capacidad de generalización, llamados de realismo experimental, que vinculan contenidos mediáticos con mediciones de opinión para generar indicadores de consumo de cierto tipo de encuadres. Tomando este procedimiento como referente, el artículo presenta los resultados sobre el impacto del consumo de contenido informativo, enfocado ya sea desde la estrategia electoral o bien en las propuestas programáticas, en el desarrollo del compromiso político de los ciudadanos durante la campaña presidencial mexicana de 2018. Los resultados muestran un importante efecto del consumo del encuadre de asunto político sobre el compromiso ciudadano en todos los indicadores medidos.

\section{Palabras clave}

Campañas electorales; Consumo mediático; Compromiso político; Encuadres noticiosos; Encuadre; Framing; Efecto framing; Realismo experimental; Actitudes políticas; Comportamientos políticos; Sofisticación política; Participación política; Comunicación política.

\section{Financiación}

Esta investigación fue financiada con los proyectos del Consejo Nacional de Ciencia y Tecnología (Conacyt) "Análisis de la cobertura mediática de las campañas electorales y su impacto en la desafección política y el compromiso democrático ciudadano" (Clave 280739) e "Infoentretenimiento político, encuadres periodísticos y desconfianza ciudadana" (Clave 256670), así como un apoyo de la Secretaria de Educación Pública de México (SEP-Prodep)

\section{Agradecimientos}

Los autores agradecen a los colaboradores del equipo de investigación su apoyo en la codificación de las noticias analizadas, así como a los revisores anónimos por sus valiosas sugerencias para mejorar este artículo. 


\begin{abstract}
International literature demonstrates the influence of news media on the political attitudes and behaviors of citizens, stemming from the coverage and framing of politics. In the context of election campaigns, this news framing effect has usually been analyzed based on experimental designs, mainly through the manipulation of strategic game and issue frames. However, the need to conduct studies with greater realism has recently been raised, to increase the external validity and generalization of the findings. This approach, called experimental realism, seeks to link media content with opinion measurements to generate consumption indicators of certain types of news frames. Taking this procedure as a reference, this paper presents results on the impact of informative content consumption, focused on either the electoral strategy or programmatic proposals, in the development of the political engagement of citizens during the 2018 Mexican presidential campaign. The findings reveal an important effect of issue frame consumption on citizen political engagement according to all the measured indicators.
\end{abstract}

\title{
Keywords
}

Election campaigns; Media consumption; Political engagement; News frames; Framing; Framing effects; Experimental realism; Political attitudes; Political behaviors; Political sophistication; Political participation; Political communication.

\section{Introducción}

Las democracias funcionales o de mayor calidad gozan de una ciudadanía comprometida democráticamente. Para que ello sea efectivo, se requiere que los ciudadanos cuenten con el interés y la motivación para informarse sobre los aspectos políticos que le rodean, así como la información suficiente que les habilite para tomar decisiones, es decir, que sostengan niveles aceptables de sofisticación política (Dalton, 2006). Ello se traduce en un mayor involucramiento en las diferentes acciones que se desempeñan en la esfera pública, así como en el desarrollo de una efectiva participación política y cívica, es decir, la existencia de una ciudadanía que mantiene un compromiso democrático fuerte (Delli-Carpini, 2004).

Si bien son numerosos los factores que pueden determinar el aumento de este compromiso ciudadano con la política, la influencia de los medios informativos y los contenidos políticos que producen -y que los ciudadanos consumen-, ha demostrado un impacto significativo en la generación y/o reforzamiento de actitudes y comportamientos políticos de los ciudadanos (Avery, 2009; De-Vreese, 2005, 2012; Gerth; Siegert, 2012). Aunque esta influencia se puede manifestar en cualquier contexto, es durante las campañas electorales cuando tiende a aumentar (Muñiz, 2015; Schuck; Boomgaarden; De-Vreese, 2013), debido a la amplia y sostenida cobertura que los medios hacen de la misma y al incremento de la necesidad de cognición que se establece entre buena parte de la ciudadanía para tomar decisiones electorales (Matthes, 2012). Es durante dichos ambientes informativos que se ha investigado, y verificado, el efecto del consumo mediático en la configuración de las cogniciones, actitudes y comportamientos del ciudadano en el terreno electoral.

\section{La influencia de los contenidos políticos de los medios ha demostrado un im- pacto significativo en la generación y/o reforzamiento de actitudes y comporta- mientos políticos de los ciudadanos}

Los estudios característicos de esta línea examinan efectos a partir del consumo de contenidos políticos genéricos, sin tomar en cuenta la manera en que las amplias diferencias en los tratamientos periodísticos de la política podrían modular dichos efectos. En respuesta a esta problemática, los autores han recurrido a la teoría de framing (encuadre) para especificar los efectos de ciertos tratamientos noticiosos sobre las actitudes ciudadanas, sobre todo desde diseños experimentales (Aalberg; Strömbäck; De-Vreese, 2012; Dimitrova; Strömbäck, 2012; Schuck et al., 2013; De-Vreese; Semetko, 2002). Estos estudios parten del supuesto de que los medios tienden a presentar la campaña desde un tratamiento informativo determinado (framing), ya sea centrado en las estrategias seguidas en la campaña electoral, así como la competencia entre los partidos y candidatos (encuadre de juego estratégico), o bien a partir de las propuestas de campaña y el debate sobre cómo éstas solucionan, o no, las problemáticas sociales (encuadre de asunto político) (Aalberg et al., 2012; Dimitrova; Stromback, 2012; Muñiz; Saldierna; Marañón, 2018; Rhee, 1997). Se asume que el consumo de información enfocada desde estos dos tratamientos informativos puede tener efectos diferenciados en la audiencia, debido a la naturaleza específica de una y otra cobertura (Aalberg et al., 2012).

La línea de investigación centrada en los efectos de los encuadres, conocida como framing effects (De-Vreese; Boomgaarden; Semetko, 2011; Druckman, 2001), ha producido resultados ambivalentes respecto al sentido de la modificación de actitudes políticas. Dentro de estos estudios destaca el trabajo pionero de Cappella y Jamieson (1997), que evaluaron el impacto de la cobertura estratégica frente a la temática (o de asunto político, en los términos utilizados en este artículo), demostrando que la cobertura desde un enfoque estratégico genera desconfianza a priori en los ciudadanos, o cinismo político, algo que ha sido confirmado por estudios posteriores (Rhee, 1997; Valentino; Beckmann; Buhr, 2001). Sin embargo, también se han detectado efectos positivos de la exposición a contenidos mediáticos enfocados desde el encuadre de asunto político, que generan una disminución del cinismo e incluso aumento de la confianza, interés en la política e involucramiento ciudadanos (De-Vreese; Semetko, 2002; Rinke; Wessler; Löb; Weinmann, 2013). 
Esta ambivalencia en los estudios pudiera estar relacionada con las limitaciones metodológicas de sus procedimientos. Al respecto, el análisis de la influencia que el framing político tiene en la audiencia ha sido desarrollado primordialmente desde acercamientos experimentales, que presentan diferentes encuadres mediante noticias manipuladas deliberadamente, a manera de estímulo, para registrar el modo en que afectan las cogniciones, actitudes o comportamientos de los participantes (De-Vreese et al., 2011; Elenbaas; De-Vreese, 2008; Entman, 1993; Matthes, 2008). Sin embargo, el poco realismo del que adolecen los diseños experimentales, aunado a la baja validez externa de estos estudios, imposibilita transferir o generalizar los efectos registrados al común de la población. Esto ha llevado a varios autores a plantear la necesidad de realizar estudios con mayor realismo, para atenuar estos problemas (De-Vreese, 2012; Shehata, 2014). Es así como, mediante un nuevo acercamiento metodológico, denominado realismo experimental, se busca vincular contenidos mediáticos con mediciones de opinión (Schuck; Vliegenthart; De-Vreese, 2016; Geers; Bos; De-Vreese, 2017), con el objetivo de generar indicadores de exposición real a contenidos concretos entre la ciudadanía, y utilizarlos como variables predictoras de sus actitudes y comportamientos políticos (Boomgaarden; Kritzinger, 2017).

Tomando estos precedentes como referencia, el presente artículo analiza el impacto de la exposición a contenido informativo de estrategia electoral (encuadre de juego estratégico) o de propuestas programáticas (encuadre de asunto político) en el desarrollo del compromiso político de los ciudadanos. Para ello, se utiliza como proxy de este compromiso político ciertas actitudes y comportamientos constitutivos del mismo, como son el interés y el conocimiento, que determinan el nivel de sofisticación política del ciudadano a nivel factual y electoral (Muñiz; Echeverría; Rodríguez-Estrada; Díaz-Jiménez, 2018), y la participación política online y offline que definen el nivel de involucramiento de la ciudadanía (Gil de Zúñiga; Molyneux; Zheng, 2014). A este fin, se trabajó con datos provenientes de una encuesta a partir de un diseño panel, realizada con una muestra amplia de la población mexicana, aplicada durante la campaña presidencial mexicana de 2018. En concreto, se levantó una ola durante la propia campaña electoral y otra en un momento posterior a las elecciones.

Por otra parte, se analizó una muestra de las noticias de los principales periódicos escritos y los noticieros televisivos matutinos y nocturnos nacionales, para detectar la presencia de los dos encuadres noticiosos con los que se trabajó el cálculo de los indicadores de exposición mediática ya descritos. La vinculación de ambos estudios permitió determinar la influencia que el consumo de alguno de estos encuadres presentes en los contenidos informativos durante la campaña electoral, ya sea el de juego estratégico o el de asunto político, tuvo en el compromiso político ciudadano.

\section{Compromiso político y medios de comunicación}

No cabe duda de que el papel que juega la ciudadanía en el desarrollo del sistema democrático es crucial, a través de su involucramiento en las acciones vinculadas al sistema político y social. Normativamente, se asume que la existencia de una sociedad civil activa y participativa, que se involucra a través de los diferentes mecanismos de participación ciudadana existentes, logrará asentar el entramado institucional, fortalecer el sistema y promover el control de la actividad de los representantes políticos (Buendía; Somuano, 2003). Una condición previa a este involucramiento es que los ciudadanos cuenten con la información necesaria para desarrollar un adecuado conocimiento sobre el sistema político y lo que en el mismo se debate y decide, y que ello pueda ser utilizado para tomar decisiones electorales (Dalton, 2006). Esto supone un escenario en el que la presencia de ciudadanos más sofisticados, políticamente hablando, desembocará en una democracia más estable (Dimitrova; Strömbäck, 2012).

Ahora bien, una dinámica de participación reducida y tan sólo reactiva a eventos electorales, desemboca únicamente en la legitimación de una democracia electoral, más no estimula el desarrollo de un sistema democrático más profundo (Buendía; Somuano, 2003). Para ello, en cambio, se necesita una ciudadanía más participativa, es decir, involucrada en las diferentes acciones que el propio sistema provee, como por ejemplo el asociacionismo, la militancia en organizaciones políticas o el desarrollo de acciones no convencionales como la manifestación o la protesta, desde una posición crítica y reflexiva. Se requiere, en suma, que este involucramiento conlleve la existencia de una ciudadanía comprometida democráticamente, tanto a nivel cívico como político, que vaya más allá de la participación electoral a través del mantenimiento de un interés y conocimiento aceptable de los aspectos políticos (Delli-Carpini; Keeter, 1996; Delli-Carpini, 2004).

Si bien existen diferentes planteamientos acerca de lo que se debe entender por compromiso político (Díaz-Jiménez; Muñiz, 2017), en términos generales se asume que éste se compone por, al menos, niveles aceptables de conocimiento y participación políticas por parte del ciudadano (Delli-Carpini; Keeter, 1996). En todas las conceptualizaciones propuestas sobre el compromiso subyace la visión de la participación política como elemento crucial para lograr una ciudadanía 
realmente comprometida (Delli-Carpini, 2004). La participación implica el grado en que el ciudadano se involucra, a través de diferentes acciones o comportamientos, en la mejora de la calidad de la vida pública de él mismo y/o de los demás (Valenzuela; Park; Kee, 2009). Esta se puede desarrollar en dos grandes planos: el cívico y el político. El primero implica llevar a cabo acciones que buscan resolver problemas mediante métodos ajenos a las elecciones y el gobierno (Delli-Carpini, 2004), mientras que la segunda conlleva el desarrollo de acciones que buscan incidir en la selección de representantes electos y el desarrollo, implementación o aplicación de políticas públicas. Ambas se pueden efectuar tanto en un terreno presencial o físico (offline), como en uno digital u online (Gil de Zúñiga et al., 2014).

En cualquier caso, para que dicha participación tenga lugar es necesario que el ciudadano cuente con un aceptable conocimiento sobre los asuntos que se presentan en la esfera pública. Se asume que un ciudadano comprometido posee opiniones estables, consistentes e informadas sobre los principales hechos acaecidos en la esfera política (Delli-Carpini, 2004). El concepto de conocimiento está íntimamente relacionado con el de sofisticación política, que define el nivel de compromiso afectivo o interés y de compromiso cognitivo, es decir, el conocimiento de los ciudadanos en relación con los asuntos políticos (Muñiz; Echeverría et al., 2018). Si bien la sofisticación política habitualmente se entiende como la conciencia política de los ciudadanos (political awareness), que define un conocimiento de corte factual sobre las reglas de funcionamiento del sistema o sus actores y actividades, también implica la existencia de una conciencia de campaña o electoral (campaign awareness), la que constituye la sofisticación política electoral (Schuck et al., 2013).

A los dos componentes básicos de participación y sofisticación políticas ya citadas, la bibliografía científica internacional aúna otros factores. Por ejemplo, Putnam (1993) considera que se requiere desarrollar actitudes de igualdad política, tolerancia, solidaridad y confianza. Por su parte, Norris (2002) incluye aspectos como el desarrollo de un capital social, el apoyo al sistema mediante la eficacia política y confianza, y el activismo político. En un intento de sintetizar las diferentes propuestas, Yung y Leung (2014) plantean que se puede desarrollar un compromiso político en tres niveles. En un primer nivel se encuentra el compromiso cognitivo, que implica estar informado sobre política, así como desarrollar una sofisticación política adecuada. Un segundo nivel es el compromiso expresivo, que implica trasladar los puntos de vista personales sobre los asuntos públicos a círculos sociales más o menos cercanos. En este terreno, por tanto, se situaría la conversación política. Finalmente, se presenta el nivel del compromiso de acción, que implica la participación a nivel institucional o convencional, como puede ser el voto o la militancia en partidos, y la no institucional, como la intervención en huelgas o manifestaciones.

La academia ha explorado estas influencias a través de una importante línea de investigación que se centra en analizar la relación entre la cobertura mediática de la política y los resultados que la misma tiene en el desarrollo de actitudes y comportamientos políticos entre los ciudadanos, una veta de estudio con abundantes trabajos desde los años 70 del pasado siglo (De-Vreese, 2005; Muñiz; Maldonado, 2011; Rojas, 2006; Schuck et al., 2013). Generalmente, esta relación se ha abordado desde dos paradigmas diferentes: el del malestar mediático (media malaise), que afirma la existencia de un efecto negativo de la exposición a medios que redunda en la generación de desconfianza, alienación y desinterés entre la audiencia con respecto a la política (Avery, 2009), y el de la movilización política (political mobilization) que plantea una visión favorable acerca de los efectos mediáticos en el terreno político (Díaz-Jiménez; Muñiz, 2017). Aunque ambas posturas plantean efectos que, en términos generales, podrían ser tenidos como contradictorios, en la realidad deben ser vistos como complementarios, pues se refieren a diferentes mecanismos de influencia (positiva vs. negativa) que actúan de forma simultánea (Shehata, 2014).

\section{Efectos del framing}

Desde que el postulado teórico del framing fuera introducido en el terreno de las ciencias de la comunicación, éste se ha convertido en una de las fuentes teóricas más ricas y destacadas dentro de los estudios en comunicación política (De-Vreese, 2012; Matthes, 2012; Rinke et al., 2013). Ello se debe a que la teoría del framing permite no sólo estudiar los contenidos de los medios, sino también evaluar el impacto que tiene el consumo mediático sobre las actitudes y comportamiento políticos De-Vreese et al., 2011; Elenbaas; De-Vreese, 2008; Entman, 1993; Matthes, 2008).

El efecto que el tratamiento informativo (framing effects) tiene sobre la opinión pública corresponde predominantemente a un proceso cognitivo, por el que los medios ayudan al establecimiento de los encuadres en los pensamientos de los individuos, al canalizar o trasladar aquellos que se originan en los medios a la mente del público (Druckman, 2001). Esto implica un proceso encadenado de recepción de la información, integración de los frames en el conocimiento del ciudadano y, a partir de estos encuadres adquiridos, alteración de las respuestas de la audiencia en cuanto a la construcción del discurso y elaboración de juicios (De-Vreese et al., 2011; Rhee, 1997).

Sin embargo, el efecto de los encuadres utilizados para ofrecer un tratamiento particular de los asuntos en las noticias no se agota en el terreno cognitivo, puesto que también comprende efectos subsecuentes en los juicios, actitudes, opiniones, emociones y/o decisiones de los individuos (Scheufele, 2006). Así, se asume que, a partir del impacto cognitivo, los encuadres tienen la capacidad de producir cambios en los juicios, actitudes, opiniones, emociones y/o decisiones de los individuos. Dentro de los estudios de framing vinculados con política destaca la línea abierta por Cappella y Jamieson (1997), quienes plantearon que el tratamiento informativo de la política, especialmente durante las campañas electorales, puede generar efectos negativos en la ciudadanía, como la desconfianza y el cinismo sobre la política y los 
políticos, lo cual puede erosionar el compromiso democrático y reducir la participación electoral de la ciudadanía (Cappella; Jamieson, 1997). Una propuesta enmarcada, por tanto, dentro del paradigma teórico del malestar mediático.

Quizá uno de los puntos de mayor interés del trabajo realizado por Cappella y Jamieson (1997) es el énfasis que ambos autores ponen en el efecto, no tanto del consumo o exposición mediática en sí, sino de ciertos enfoques o encuadres (news frames) utilizados para tratar informativamente la política. Al referirse a los encuadres políticos, Matthes (2012) señala que éstos son conjuntos o

"paquetes de argumentos temáticos consistentes, originalmente propuestos por opositores y defensores"

en una campaña o debate (p. 254). Así pues, se asume la posibilidad de que los encuadres provengan, tanto de los actores políticos, como de los periodistas al reinterpretar la información que es aportada en los discursos políticos. Aunque son muchos los encuadres que los medios utilizan para presentar la política, en la investigación en comunicación política ha dominado el planteamiento de la existencia de un enfoque de juego estratégico frente a un tratamiento de asunto político; ambos encuadres, planteados originalmente por Cappella y Jamieson (1997), son considerados como prototípicos dentro de la información sobre campañas electorales y otro tipo de asuntos referidos a la política (De-Vreese, 2012; De-Vreese; Semetko, 2002;

\author{
En la investigación en comunicación po- \\ lítica ha dominado el planteamiento de \\ la existencia de un enfoque o framing de \\ juego estratégico frente a un tratamien- \\ to de asunto político
} Schuck et al., 2013).

En cuanto al encuadre de juego estratégico (strategy game frame), es utilizado por los medios para presentar una cobertura enfocada en la estrategia y tácticas de los contendientes, su posición en las encuestas de opinión, en quién gana y quién queda atrás, así como otros aspectos que significan a la campaña como una "carrera de caballos" (Dimitrova; Stromback, 2012). En este planteamiento del encuadre se observa una fusión de diferentes conceptos, tradicionalmente estudiados por separado. Por una parte, se trabaja con el encuadre de juego (game frame), que tiende a dar importancia a los datos de encuestas, presentar la política como un asunto de ganadores y vencedores o utilizar metáforas bélicas (De-Vreese, 2005). Por otra parte, el encuadre de carrera de caballos (horse race frame), que prioriza la competición entre los candidatos en búsqueda de la victoria final (Shehata, 2014). Finalmente, el encuadre estratégico (strategic frame), que se centra en el estilo del político, sus acciones y motivaciones (Rhee, 1997). Muchas veces estas definiciones se han percibido como cercanas, vinculadas e incluso intercambiables, por lo que actualmente tienden a verse como dimensiones de un mismo encuadre más extenso denominado de juego estratégico (strategic game frame) (Aalberg et al., 2012; De-Vreese, 2005; De-Vreese; Semetko, 2002).

Frente a este encuadre, en las noticias sobre política también suele manifestarse un encuadre de asunto político (issue frame) que, en palabras de Rhee (1997), es utilizado por los medios para comunicar

"propuestas a los problemas planteados, información sobre quién está contribuyendo con estas alternativas y las consecuencias de los problemas y las propuestas planteadas" (p. 30).

Es decir, la información encuadrada desde este frame tiende a enfatizar los asuntos políticos sustantivos y políticas públicas, así como las propuestas que realizan los candidatos y/o partidos políticos tanto en campañas electorales como en otros periodos, así como la discusión pública que se realiza acerca de las implicaciones de dichas propuestas (Aalberg et al., 2012; Dimitrova; Stromback, 2012; Muñiz; Saldierna; Marañón, 2018). Ahora bien, aunque este tratamiento pareciera estar más cerca del ideal normativo de cobertura noticiosa durante una campaña política, al proporcionar a la ciudadanía información a partir de la cual puedan construir conocimiento político y formular sus decisiones electorales, la investigación realizada en diferentes contextos nacionales demuestra que la cobertura informativa de las campañas prioriza el uso del juego estratégico (Aalberg et al., 2012; Muñiz, 2015; Schuck et al., 2013).

Aunque pueden presentarse otros encuadres en la cobertura de la política (Muñiz; Saldierna; Marañón, 2018), el estudio de los de juego estratégico y de asunto político han centrado buena parte de los debates académicos y públicos acerca del papel de los medios de comunicación durante las campañas electorales, así como su influencia en las actitudes y comportamientos de los ciudadanos (Schuck et al., 2013). Sin duda, la línea de estudio más fuerte del efecto del framing (framing effect) en el terreno político se ha centrado en el papel del tratamiento de juego estratégico vs. de asunto político en la disminución o aumento de este cinismo. En este sentido, muchos de los estudios realizados en esta línea han encontrado vinculaciones entre la exposición a noticias con tratamiento de juego estratégico y el cinismo político (Aalberg et al., 2012; De-Vreese, 2005; De-Vreese; Semetko, 2002, Rhee, 1997; Schuck et al., 2013; Valentino et al., 2001). Los datos del estudio de Cappella y Jamieson (1997) mostraron que el tratamiento estratégico genera en los ciudadanos desconfianza, algo que no sucedía con el encuadre de asunto político. Un resultado también corroborado por Valentino et al. (2001) o Rhee (1997) respecto del impacto del encuadre de juego estratégico en un aumento de reacciones políticas negativas entre los ciudadanos -disminución en el conocimiento, confianza y compromiso políticos-, que a final de cuentas puede ir en detrimento de la democracia.

Sin embargo, no todos los investigadores están de acuerdo con que se produzca este efecto negativo del framing (Aalberg et al., 2012; Rinke et al., 2013). Aunque el encuadre de juego sí puede producir efectos negativos en el incremento 
del cinismo y la disminución del interés político (Shehata, 2014), la información estratégica sobre encuestas y sondeos e incluso la relativa a la confrontación entre políticos, estimula la atención a la cobertura política e incluso la participación política (De-Vreese; Semetko, 2002), ya que el enfoque estratégico es más emocionante para la audiencia. Al respecto, se ha señalado que este tipo de información no necesariamente hace reducir la participación política de la ciudadanía, sino que puede estimularla (De-Vreese; Semetko, 2002). Por su parte, hay menos estudios sobre el efecto framing respecto al compromiso y la movilización política de la ciudadanía. Al respecto, el estudio de Shehata (2014) detectó que la exposición al encuadre de asunto político genera una disminución de cinismo y un aumento de la confianza e interés en la política, indicadores necesarios del compromiso político.

\section{Realismo experimental en el estudio del efecto framing}

Si bien el proceso framing o el proceso de tratamiento implica una primera fase donde se crean los encuadres y éstos son traslados a la ciudadanía a través, normalmente, de los medios, no se puede olvidar la última fase del proceso, que se circunscribe al efecto que estos encuadres trasladados tienen en la audiencia que los recibe (De-Vreese et al., 2011; Elenbaas; De-Vreese, 2008; Entman, 1993; Matthes, 2008). Los estudios de framing effect exploran cómo y bajo qué circunstancias los encuadres utilizados para elaborar los mensajes afectan las respuestas de la audiencia (De-Vreese $e t$ al., 2011). En el contexto de las campañas electorales, son numerosos los estudios realizados para analizar el impacto los frames políticos presentes en las noticias sobre las actitudes ciudadanas, principalmente el efecto de los citados "juego estratégico" y “asunto político" (Schuck et al., 2013; De-Vreese; Semetko, 2002).

En su mayoría, estos estudios han sido desarrollados desde metodologías de corte experimental, buscando el estudio de la causalidad a partir de un mayor control del diseño de investigación (De-Vreese, 2012). Sin embargo, y a pesar de la importante evidencia que esta línea de investigación ha generado, no deja de haber dudas sobre la posibilidad de trasladar los resultados de los estudios realizados de forma aislada al ámbito real de las audiencias, debido a la baja validez externa de estos diseños (Shehata, 2014). Al respecto, se ha cuestionado el hecho de que los experimentos fuerzan a los participantes a exponerse a contenidos, a menudo en situación de laboratorio. Ello crea un contexto no realista, que no refleja la relación cotidiana de la audiencia con los medios, no captura las variaciones individuales del consumo mediático, y supone una situación artificial frente a la cobertura periodística real de la política y las campañas electorales (De-Vreese, 2012).

A este problema se aúnan los inherentes a las medidas típicas utilizadas para medir el consumo mediático (Geers et al., 2017). Normalmente, el diseño de instrumentos obliga a confiar en las respuestas dadas por los participantes sobre consumo mediático, que pueden estar sesgadas por la deseabilidad social. Junto a ello, los diseños experimentales normalmente trabajan con instrumentos reducidos, buscando obtener la mayor validez interna posible. Ello hace que el diseño del cuestionario pueda llegar a reducir la variedad de preguntas de consumo mediático, limitándolo a unas consideradas principales, que no agotan la complejidad del consumo realizado por la audiencia. Esta situación, además, lleva a que en muchas ocasiones exista una falta de medición de consumo de espacios específicos de los medios, por privilegiar el uso de escalas más generalistas de atención específica de contenidos y, sobre todo, de exposición genérica a los diferentes medios de comunicación.

Todo ello supone una limitación para este tipo de estudios experimentales, sobre todo cuando desde hace años se viene planteando en la bibliografía de comunicación política la necesidad de realizar estudios con mayor realismo acerca del impacto que el consumo de contenidos de los medios de comunicación tiene en el desarrollo de actitudes, cogniciones y comportamientos políticos (De-Vreese, 2005; Geers et al., 2017). El realismo experimental es una respuesta a estas inquietudes científicas (De-Vreese, 2012; Matthes, 2008; Shehata, 2014). Como diseño metodológico, busca vincular mediciones de la opinión pública con una evaluación más realista del consumo de contenidos de los medios -es decir, a la cobertura ofrecida a asuntos o momentos como las campañas electorales- a fin de aumentar la validez externa y generalización de los estudios abocados a comprender el impacto real que la cobertura mediática está teniendo en el desarrollo de las actitudes y comportamientos de la ciudadanía (Geers et al., 2017; Shehata, 2014; Schuck et al., 2016).

Dicho diseño se ha utilizado en numerosos trabajos en los estudios de framing, en los cuales se generan indicadores de consumo de contenidos encuadrados de cierta manera (De-Vreese, 2012; Shehata, 2014). El procedimiento consiste en ponderar la medición de uso mediático -registrado a través de la etapa inicial de una encuesta panel-con los valores de presencia del contenido concreto en medios, en este caso encuadres, detectados a partir del análisis de contenido. A partir de esta vinculación se generan indicadores de exposición a contenidos (Boomgaarden; Kritzinger, 2017) que permiten superar las limitaciones de mediciones más sencillas de consumo a través de encuestas, que no toman en cuenta la influencia del contenido real consumido en el período de tiempo estudiado por parte del encuestado. Posteriormente, estos nuevos índices de consumo de contenidos particulares se correlacionan con las medidas de actitudes políticas obtenidas en etapas posteriores de la encuesta panel. Con ello, se puede además evaluar si estas variables de consumo generan algún cambio de actitudes en el tiempo (Schuck et al., 2016; Boomgaarden; Kritzinger, 2017; Geers et al., 2017).

La investigación sobre comunicación política ha marcado claramente la existencia de una exposición diferenciada a medios y contenidos, lo que genera resultados distintos en función de si se trabaja con medidas de exposición genérica a medios o de atención a contenidos concretos de esos medios (Holtz-Bacha, 1990; Muñiz; Maldonado, 2011). Al respec- 
to, se ha detectado normalmente un mayor efecto provocado por la atención a contenidos concretos de política que un seguimiento genérico de los medios (Rojas, 2006). El realismo experimental contribuye, sin duda, a mejorar el análisis de este efecto, al suponer un diseño que permite crear indicadores más precisos de medición de la atención al tratamiento informativo realizado de la política en los medios.

Son ya varios los estudios que se pueden localizar en la bibliografía internacional que utilizan este diseño para evaluar la influencia del consumo de encuadres sobre diferentes actitudes y comportamientos (De-Vreese, 2012). Por ejemplo, en su estudio Schuck et al. (2016) detectan que el consumo de conflicto en medios movilizaba a los ciudadanos a votar. Por su parte, Wettstein (2012) detecta un efecto de traslación de la saliencia de los frames durante un referéndum, de tal suerte que el consumo de encuadres determina la interpretación que hace el público de los asuntos debatidos en el proceso electoral, quedando patente el efecto de canalización cognitiva de los frames.

En relación con el efecto del consumo de encuadres relativos a la presentación de la política como juego estratégico o como asunto, también se identifican varios estudios que han trabajado con diferentes variables dependientes (Shehata, 2014). Es el caso, por ejemplo, del trabajo realizado por Geers et al. (2017), quienes evaluaron la influencia del consumo de noticias de asunto político frente al consumo de información de encuestas, un componente del encuadre más amplio de juego estratégico, sobre la volatilidad electoral, traducida bien en conversión o bien en cristalización de opciones electorales. Los autores detectan que el consumo de asunto político en prensa incrementa la cristalización del voto, mientras que el consumo de encuestas en prensa hace que ésta disminuya. Por su parte, el consumo de contenidos de encuesta en televisión aumenta la conversión del voto, mientras que el de asunto político en televisión hace que ésta disminuya.

Pero quizá el estudio que más se ajusta al que se presenta en este artículo es el realizado por Shehata (2014), en el que evaluó el impacto del consumo del encuadre de juego, en contraposición al consumo de asunto político, sobre el cinismo y compromiso políticos desarrollados por los ciudadanos. La investigación demuestra que la exposición al tratamiento de juego tendía a hacer aumentar el cinismo político mantenido por los participantes en la encuesta. Por el contrario, consumir contenidos informativos enfocados desde el asunto político tendía a presentar una influencia más positiva en ciertas actitudes políticas de los ciudadanos, en este caso tanto en la confianza, como el interés político.

Como se ha podido constatar, los antecedentes proceden de otros contextos diferentes al mexicano, donde no existe aún evidencia del uso de esta metodología. Por ello, y tomando con referencia estos antecedentes teóricos, se trabajó con la campaña electoral presidencial mexicana de 2018 como caso de estudio para dar respuesta a las siguientes preguntas de investigación acerca de la influencia del consumo de frames en el compromiso político, manifestado a través de las diferentes actitudes y comportamientos políticos estudiados:

PI1: ¿En qué medida el consumo de información con tratamiento de juego estratégico y de asunto político impactó en el nivel de sofisticación política factual?

PI2: ¿En qué medida el consumo de información con tratamiento de juego estratégico y de asunto político impactó en el nivel de sofisticación política electoral?

PI3: ¿En qué medida el consumo de información con tratamiento de juego estratégico y de asunto político impactó en el nivel de participación política offline?

PI4: ¿En qué medida el consumo de información con tratamiento de juego estratégico y de asunto político impactó en el nivel de participación política online?

\section{Método seguido}

\subsection{Estudio primero: Análisis de contenido de noticias de televisión}

En primer lugar, y atendiendo al objetivo de la investigación que busca generar indicadores más exactos sobre consumo mediático, se realizó un análisis de contenido de las noticias emitidas en prensa y televisión durante la campaña electoral a la presidencia de México de 2018. Para ello, se analizaron las noticias publicadas en los periódicos Reforma $(N=343$, $19.47 \%)$, El universal $(N=158,8.97 \%)$, Excelsior $(N=210,11.92 \%)$, Milenio $(N=182,10.33 \%)$ y La jornada $(N=220$, $12.49 \%)$. Asimismo, se analizaron los informativos matutinos y nocturnos de Televisa $(N=227,12.88 \%)$, TVAzteca $(N=$ $181,10.27 \%)$ e Imagen TV ( $N=241,13.68 \%)$. Se optó por trabajar con estos medios debido a que, aunque paulatinamente los nuevos medios o medios sociales han ido ganando espacio en la obtención de información de campaña para buena parte de la ciudadanía, la prensa y, sobre todo, la televisión, se mantienen como importantes medios usados en México para seguir el desarrollo de las campañas electorales, así como para establecer su agenda política (Muñiz, 2015).

El corpus de noticias se determinó a partir de un muestreo aleatorio sistemático de los tres meses de campaña electoral, en dos fases diferenciadas. En la primera, que abarcó los meses de marzo, abril y mayo, se seleccionaron todas las noticias publicadas cada cuatro días de campaña, comenzando el 30 de marzo y terminando el 31 de mayo. En la segunda fase, que transcurrió durante el mes de junio de 2018, se decidió levantar una muestra a partir de seleccionar todas las noticias emitidas en las televisoras cada dos días, desde el 1 de junio y finalizando el 28 de junio. Únicamente se revisaron los días de diario, es decir, de lunes a viernes, atendiendo a que los fines de semana no se emitían informativos de televisión. El proceso de búsqueda dio como resultado la detección de 1762 notas informativas o unidades de análisis, 
1113 en prensa (63.17\%) y 649 (36.83\%) en televisión (de las que 365 se emitieron en noticieros de la mañana y 284 en informativos de la noche).

El libro de códigos quedó compuesto por dos grandes variables que fueron evaluadas en las noticias: el encuadre de juego estratégico y el encuadre de asunto político. Para ello, se siguieron las propuestas metodológicas de autores como Aalberg et al. (2012), Cappella y Jamieson (1997), Dimitrova y Strömbäck (2012), Elenbaas y De-Vreese (2008) o Muñiz, Saldierna y Marañón (2018), relativas a la operacionalización y medición de ambos encuadres noticiosos.

En relación con el encuadre de juego estratégico, se utilizó una escala de cinco reactivos para medir la presencia del encuadre a partir de evaluar, de forma dicotómica $(1=$ sí; 0 = no), si el texto de la nota etiquetaba

"a los políticos o partidos como ganadores y/o perdedores en elecciones, debates legislativos, negociaciones gubernamentales o en asuntos políticos en general",

si mencionaba

"estrategias de políticos o partidos para ganar elecciones, negociaciones o debates temáticos", si señalaba

"las implicaciones o consecuencias para los políticos o partidos de las elecciones, negociaciones gubernamentales, debates legislativos u otros eventos de corte político", si aportaba

"datos de opinión, encuestas y/o posición de la opinión pública y ciudadanía hacia los políticos, partidos, campaña electoral, asuntos, etc."

y si utilizaba

"metáforas o expresiones generalmente asociadas con el deporte, competencia o incluso la guerra".

La consistencia interna de la escala fue baja tanto para televisión $(\alpha=.56)$ como para prensa $(\alpha=$ .43), en consonancia con estudios previos (Muñiz, 2015; Muñiz; Saldierna; Marañón, 2018). Un ejemplo de este tipo de encuadre noticioso se puede encontrar en la noticia publicada el 28 de junio de 2018 en el periódico Reforma. En la misma, el periódico titulaba "Abarrota el Azteca" para referirse al acto de cierre de campaña de Andrés Manuel López Obrador (figura 1).

En cuanto al encuadre de asunto político, también se utilizó una escala de cinco reactivos para medir la presencia del encuadre a partir de evaluar, de forma dicotómica (1 = sí; 0 = no), si el texto de la nota señalaba

"problemas del mundo real, situaciones o procesos que tienen implicaciones políticas explícita o implícitamente",

si abordaba

"problemas y/o soluciones sobre ciertas propuestas políticas, políticas públicas, sobre legislación, propuestas legislativas, etc.",

si señalaba

"la postura y en su caso, las declaraciones de los políticos acerca de ciertas propuestas políticas, políticas públicas, sobre legislación, propuestas legislativas, etc.",

si abordaba

"el debate entre actores políticos acerca de problemas del mundo real, situaciones o procesos que tienen implicaciones políticas explícita o implícitamente"

y si explicaba

“las implicaciones o impactos que una legislación, propuesta legislativa, propuesta de gobierno, propuesta de campaña o política pública tiene para las personas y/o sociedad". 
La consistencia interna de la escala fue aceptable para prensa ( $\alpha=.69$ ), pero baja para televisión ( $\alpha=.59)$, aunque de nuevo con valores en consonancia con estudios previos (Muñiz, 2015; Muñiz; Saldierna; Marañón, 2018). Un ejemplo de este tipo de encuadre noticioso se puede encontrar en la noticia publicada el 26 de junio de 2018 en el periódico La jornada titulada "Equipo de AMLO presenta agenda ambiental para 20182024", donde se discute la política ambiental del candidato (figura 2).

El análisis de los datos fue realizado por diferentes colaboradores del Laboratorio de Comunicación Política ubicado en la Facultad de Ciencias Políticas y Relaciones Internacionales de la Universidad Autónoma de Nuevo León. Una vez concluido el trabajo de codificación, se hizo un nuevo análisis con una muestra de 160 noticias, 80 pertenecientes a cada medio de comunicación, para realizar un chequeo inter-jueces, que arrojó un valor .74 para prensa y .81 para televisión de acuerdo según la fórmula Alfa de Krippendorff.

\subsection{Estudio segundo: Encuesta panel durante la campaña electoral}

Por otra parte, y de acuerdo con el objetivo del estudio, se procedió a medir el nivel de compromiso político mantenido por los ciudadanos mexicanos durante la campaña electoral de 2018. Para ello, y atendiendo a que se planteó un diseño metodológico explicativo, se aplicó una encuesta analítica panel en dos momentos de la campaña. Se utilizó esta técnica por permitir analizar no sólo opiniones y actitudes de una población en un momento coyuntural, sino también determinar el cambio que se produce en las mismas a lo largo del tiempo, así como evaluar qué variables afectan en ese cambio (De-Vreese; Semetko, 2002; Boomgaarden; Kritzinger, 2017). Además, este diseño permite, en consonancia con lo planteado por los autores, generar un proceso de realismo experimental, al vincular datos de análisis de contenido con mediciones de consumo mediático.

Para realizar la encuesta se contrató a la empresa Survey Sampling International (SSI), con el objeto de trabajar con una muestra amplia de la población mexicana, que contó con un margen de error del $3 \%$ y un nivel de confianza del $98 \%$, para poder así garantizar la permanencia de un volumen importante de participantes en la segunda ola del estudio. La primera ola se realizó durante el mes de junio de 2018, con el objetivo de registrar el seguimiento de prensa e informativos televisivos para informarse de la campaña electoral. El levantamiento se realizó entre el 1 y el 25 de junio de 2018 , participando una muestra de 1516 entrevistados durante esta ola primera. La segunda aplicación se realizó una vez pasada la elección y cuando los conteos distritales para la elección presidencial quedaron cerrados, por lo que el trabajo de campo comenzó el 9 de julio y terminó el 19 de ese mes. En total participaron 1000 entrevistados en ambas olas.

De los datos obtenidos, únicamente se contó con los relativos a los participantes que fueran mayores de edad ( $\geq 18$ años) y censados como votantes en la República. En este sentido, en la muestra final $(N=1000)$ participaron ciudadanos de todas las entidades federativas de la república. Además, un $55.7 \%$ de la muestra estaba constituido por hombres ( $n$ = 557), por un $44.3 \%$ de mujeres $(n=443)$, con edades comprendidas entre 19 y 81 años $(M=44, D E=14)$. La muestra contempló participantes con diferentes niveles educativos, siendo el grupo mayoritario el correspondiente a aquellos que tenían estudios profesionales $(n=598,60.2 \%)$ o de preparatoria $(n=194,19.4 \%)$. Asimismo, un $41.4 \%$ de participantes reportaron unos ingresos mensuales de entre $\$ 10,001.00$ y $\$ 30,000.00(n=380)$, un $32.3 \%$ menores a $\$ 10,001.00$ $(n=296)$ y un $24.1 \%$ más de $\$ 30,001.00(n=241)$. A pesar de contar con una muestra amplia, que podía representar ampliamente los hábitos comunicativos, así como actitudes y comportamientos políticos mantenidos por los mexicanos durante el proceso electoral presidencial estudiado, cabe señalar que los estadísticos sociodemográficos no se ajustaron perfectamente a los parámetros poblacionales mexicanos, tomando como referencia lo señalado en la Encuesta Intercensal de 2015 del Instituto Nacional de Estadística y Geografía (INEGI) de México. Por ello, en los análisis realizados para determinar el comportamiento de las diferentes variables constitutivas del compromiso político de los ciudadanos se controló la influencia de estas variables, para eliminar su posible efecto.

En la elaboración del cuestionario aplicado en la encuesta panel se tomaron en cuenta una serie de variables que hicieran referencia al consumo mediático y las actitudes comportamientos políticos de los ciudadanos a lo largo de la campaña electoral. 
En este sentido, en la primera ola de la encuesta panel se midió el nivel de consumo de medios tradicionales realizado por los participantes para seguir los acontecimientos de la campaña electoral. Así, se preguntó cuántos días a la semana habían leído en papel los periódicos La jornada, Reforma, Excélsior, Milenio y El universal y habían visto los noticieros matutinos y nocturnos de las televisoras Las estrellas de Televisa, TV Azteca 1 e Imagen TV para enterarse de la campaña electoral presidencial, asumiendo una semana natural de siete días, por lo que la escala oscilaba entre 0 (ningún día) y 7 (todos los días).

Por su parte, las variables constitutivas del compromiso político fueron medidas en la segunda ola de la encuesta panel. Para ello, se trabajó con indicadores relativos a tres aspectos considerados habitualmente constitutivos del compromiso político (Yung; Leung, 2014): participación política, interés hacia la política y conocimiento político, estos dos últimos componentes que en conjunto determinan el nivel de sofisticación política (Muñiz; Echeverría et al., 2018; Schuck et al., 2013).

En cuanto a la sofisticación política, se trabajaron dos indicadores siguiendo la propuesta realizada por Muñiz, Echeverría et al. (2018), acerca de la existencia de una sofisticación factual y una sofisticación electoral. Con relación a la factual, se midió en primer lugar el interés político de los participantes, siguiendo la escala propuesta por Muñiz y Maldonado (2011) que se compone de cuatro ítems que miden el interés a nivel local o municipal, estatal, nacional o federal y, finalmente, internacional. Para ello, se utilizaron escalas Likert de 5 puntos que oscilaban entre nada interesado (1) y muy interesado (5) en cada uno de los niveles de la política $(\alpha=.87)$. Asimismo, se creó una escala de conocimiento político factual, a partir de la adición de las respuestas ( 1 = acertada, 0 = fallada) a cinco preguntas como, por ejemplo, ¿Qué partido tenía mayoría en la Cámara de Diputados antes de las pasadas elecciones? $(K R-20=.16)$. Con ambas variables se creó el indicador de sofisticación política, dando doble peso al componente cognitivo frente al afectivo, que oscila entre 1.75 para reflejar la máxima sofisticación política y 0.25 para reflejar la mínima.

En relación con la sofisticación política electoral, se siguió la misma propuesta que para el indicador anterior. En primer lugar, se midió el interés demostrado por los ciudadanos hacia las elecciones a la Presidencia de México mediante una escala Likert de 5 puntos que oscilaba entre estar nada interesado (1) y encontrarse muy interesado (5). Además, se midió el nivel de conocimiento político electoral de los participantes mediante una batería de cuatro preguntas, ante las que se debía indicar qué candidato a la presidencia había planteado cierta propuesta. Las respuestas fueron codificadas como acierto (1) o fallo (0), con los mismos criterios seguidos para conocimiento político factual (KR-20 = .57). Para crear el indicador se trabajó de la misma manera que respecto de la sofisticación política factual.

En cuanto a la medición de la participación política, tanto offline como online, se crearon dos escalas a partir de reactivos empleados en anteriores estudios (Gil de Zúñiga et al., 2014). Así, para medir la participación en el terreno real se preguntó a los participantes en la encuesta qué tanto ( 1 = nunca a 5 = muy frecuentemente) habían, por ejemplo, trabajado para algún candidato o partido político en campañas políticas, intentado convencer a sus amigos para que votaran por los candidatos que creían que tenían que ganar, o participado en plantones, colocación de mantas o bloqueo de calles al no estar de acuerdo con alguna decisión del gobierno $(\alpha=.93)$. En cuanto a la participación política en el terreno digital, se preguntó a los participantes si habían realizado actividades como tratar de que no votaran por un candidato a través de las redes sociales, expresar su opinión personal acerca de asuntos sociales o políticos o hacerse voluntario de la campaña política de algún candidato. Para ello se utilizó una escala de Likert similar a la participación política real ( $\alpha=.87$ ).

Finalmente, se contempló el uso de una serie de variables de control de carácter sociodemográfico. En concreto, se les cuestionó a los participantes acerca de su género ( 0 = masculino; 1 = femenino) y su edad, en años cumplidos. También se pidió que indicaran cuál era, aproximadamente, el nivel de ingresos mensual de su núcleo familiar, con una escala que oscilaba entre menos de 6,000 pesos (1) y más de 30,001 pesos (4). En último lugar, se evaluó de forma adicional el nivel de estudios de los participantes, con una escala que osciló entre no tiene (1) y estudios de posgrado (7).

\section{Análisis de los resultados}

El primer análisis de los datos se centró en determinar en qué medida fueron utilizados los encuadres noticiosos por los diferentes medios en sus noticias para tratar informativamente la campaña electoral presidencial de México en 2018. El análisis de la muestra global de noticias permitió determinar que existían diferencias a nivel estadístico en el uso de ambos encuadres, $t(1761)=4.099, p<.001, d=0.128,95 \%$ IC $[0.02,0.05]$, aunque ésta fue más bien débil atendiendo al valor del tamaño del efecto detectado (Rubin, 2010). En este sentido, el encuadre de juego estratégico tendió a utilizarse levemente más en las noticias $(M=.24, D E=.24)$ que el encuadre de asunto político $(M=.21, D E=.26)$. Además de este análisis a partir del corpus de noticias, se procedió a comparar el uso de los encuadres de juego estratégico y de asunto político en la prensa frente a la televisión, para determinar si entre ambos medios estudiados se presentaban diferencias (tabla 1).

Tabla 1. Nivel de presencia de cada encuadre noticioso en los diferentes medios

\begin{tabular}{|l|c|c|c|c|c|c|}
\cline { 2 - 6 } \multicolumn{1}{c|}{} & \multicolumn{2}{|c|}{ Prensa } & \multicolumn{2}{c|}{ Televisión } & \multirow{2}{*}{$\boldsymbol{t}(1760)$} & $\boldsymbol{p}$ \\
\cline { 2 - 7 } & $\boldsymbol{M}$ & d de Cohen & $\boldsymbol{M}$ & .20 & 16.494 & $<.001$ \\
\hline Juego estratégico & .31 & .24 & .13 & .21 & 9.884 & $<.001$ \\
\hline Asunto político & .26 & .28 & .13 & 0.506 \\
\hline
\end{tabular}

Nota: $N_{\text {total }}=1762 ; N_{\text {prensa }}=1113$ en prensa; $N_{\text {televisión }}=649$ 
En relación con el uso del encuadre de juego estratégico, se detectaron diferencias estadísticamente significativas y fuertes entre los dos medios, $t(1760)=16.494, p<.001, d=0.835,95 \%$ IC $[0.16,0.21]$. En concreto, se pudo determinar que fue la prensa el medio que en mayor medida presentó noticias donde se enfocaba la información de campaña de una manera prioritaria con el encuadre de juego estratégico $(M=.31, D E=.24)$, mientras que la televisión tendió a utilizarlo en una menor medida $(M=.13, D E=.20)$. Con respecto a la presencia del encuadre de asunto político en las noticias, también se detectaron diferencias estadísticamente significativas entre ambos medios, $t(1760)=9.884, p<.001, d=$ $0.506,95 \%$ IC $[0.10,0.15]$, si bien en este caso las mismas fueron moderadas. De nuevo, fue la prensa donde más noticias fueron enfocadas desde el asunto político $(M=.26, D E=.28)$, en comparación con la televisión $(M=.13, D E=.21)$.

Una vez determinados los niveles de presencia del encuadre de juego estratégico y de asunto en los diferentes periódicos e informativos de televisión analizados, se procedió a crear los indicadores de consumo de cada uno de estos encuadres de los participantes en la encuesta. Para ello, primero se tomaron en cuenta las mediciones de seguimiento de los diferentes periódicos e informativos de televisión realizadas en la primera ola de la encuesta panel. A partir de estos valores, se procedió a ponderar el consumo mediático siguiendo la propuesta realizada por autores en estudios previos sobre realismo experimental (Schuck et al., 2016; Geers et al., 2017), que contempla sumar las multiplicaciones del nivel de consumo de cada contenido informativo manifestado por los participantes por el nivel de presencia que tiene cada encuadre en el contenido informativo correspondiente, dividiendo el total obtenido entre el número de contenidos informativos utilizados. En este sentido, para calcular los indicadores de consumo de encuadres se utilizó la siguiente fórmula:

Consumo de encuadre $=\frac{\sum \text { Tipo de noticias }_{\text {medio }} \mathrm{X}_{\text {Exposición mediática }} \text { medio }}{\Sigma \text { Medios }}$

Con posterioridad a la creación de cada uno de los indicadores de consumo de los dos encuadres, se procedió a calcular los descriptivos de estos nuevos reactivos, que fungirían como variables independientes en el estudio, y de los reactivos constitutivos del compromiso político, que actuarían como variables dependientes del modelo (tabla 2). En cuanto al consumo de ambos encuadres noticiosos, el análisis permitió determinar la existencia de diferencias débiles a nivel estadístico, $t(999)=23.972, p<.001, d=0.109,95 \%$ IC $[0.03,0.04]$. En este sentido, aunque el consumo de ambos encuadres fue relativamente similar, se pudo determinar que hubo un mayor consumo de juego estratégico $(M=0.38, D E=0.36)$ que un consumo del encuadre de asunto político $(M=0.34, D E=0.32)$.

Tabla 2. Datos descriptivos de las variables de la encuesta panel

\begin{tabular}{|l|c|c|c|}
\hline & Ola & M & DE \\
\hline Consumo de juego estratégico & 1 & 0.38 & 0.36 \\
\hline Consumo de asunto político & 1 & 0.34 & 0.32 \\
\hline Compromiso político & & & $0-1.47$ \\
\hline Sofisticación política factual & 2 & 1.35 & 0.01 \\
\hline Sofisticación política electoral & 2 & 2.53 & 0.02 \\
\hline Participación política offline & 2 & 1.68 & $0.35-1.75$ \\
\hline Participación política online & 2 & 2.13 & $1-5$ \\
\hline
\end{tabular}

Por otra parte, también se analizó la presencia de los reactivos constitutivos del compromiso político (tabla 2), todos ellos medidos en la segunda ola de la encuesta panel. En términos generales, se observa un elevado nivel de sofisticación política factual entre los participantes en la encuesta $(M=1.35, D E=0.01)$, con un nivel cercano al límite superior, en gran medida por el elevado interés político demostrado por los participantes. Asimismo, se detectó un nivel aceptable de sofisticación política factual $(M=2.53, D E=0.02)$, al superarse también la media teórica de la escala, en gran medida por el elevado interés por lo ocurrido en la campaña demostrado por los participantes. Sin embargo, los menores niveles se detectaron con respecto a la participación política, tanto offline $(\mathrm{M}=1.68, \mathrm{DE}=0.93)$ como online $(M=2.13, D E=$ 1.06).

Con el objetivo de responder a las preguntas de investigación del estudio, relativas al impacto del consumo de encuadres sobre los indicadores de compromiso político, se procedió a realizar diferentes regresiones lineales múltiples jerárquicas con los indicadores de compromiso como variables criterio. En un primer bloque de cada regresión se introdujeron las variables de control, determinadas por el sexo, edad y nivel de estudios del participante, así como el nivel de ingresos mensual del hogar. Ello para poder determinar una medición más real acerca del peso del consumo de cada encuadre sobre las variables dependientes, al controlar previamente estas variables sociodemográficas. En el segundo bloque se introdujeron, mediante un modelo por pasos, las diferentes variables de consumo para determinar únicamente aquellas que llegaron a explicar el indicador de compromiso. 
Tabla 3. Resultados de la regresión jerárquica para la sofisticación política factual

\begin{tabular}{|c|c|c|c|c|c|c|c|}
\hline & \multirow{2}{*}{ B } & \multicolumn{2}{|c|}{$95 \%$ IC para B } & \multirow{2}{*}{ EE B } & \multirow{2}{*}{$\beta$} & \multirow{2}{*}{$R^{2}$} & \multirow{2}{*}{$\Delta R^{2}$} \\
\hline & & LI & $L S$ & & & & \\
\hline Paso 1 & & & & & & .07 & $.07^{* * *}$ \\
\hline Constante & 1.09 & 0.94 & 1.24 & 0.08 & & & \\
\hline Edad del encuestado & 0.00 & -0.01 & 0.01 & 0.01 & 01 & & \\
\hline Género del encuestado & -0.02 & -0.05 & 0.02 & 0.02 & -.04 & & \\
\hline Ingreso mensual hogar & 0.05 & 0.03 & 0.07 & 0.01 & $.20^{* * *}$ & & \\
\hline Nivel de estudios & 0.02 & 0.01 & 0.05 & 0.01 & $.07^{+}$ & & \\
\hline Paso 2 & & & & & & 12 & $.05 * * *$ \\
\hline Constante & 0.99 & 0.84 & 1.14 & 0.08 & & & \\
\hline Edad del encuestado & 0.01 & 0.01 & 0.01 & 0.01 & .05 & & \\
\hline Género del encuestado & -0.01 & -0.04 & 0.03 & 0.02 & -.01 & & \\
\hline Ingreso mensual hogar & 0.05 & 0.04 & 0.07 & 0.01 & $.20^{* * *}$ & & \\
\hline Nivel de estudios & 0.02 & -0.01 & 0.05 & 0.01 & $.07^{+}$ & & \\
\hline Consumo de juego estratégico & 0.17 & 0.13 & 0.21 & 0.02 & $.25^{* * *}$ & & \\
\hline Paso 3 & & & & & & .13 & $.01^{* * *}$ \\
\hline Constante & 0.98 & 0.84 & 1.13 & 0.08 & & & \\
\hline Edad del encuestado & 0.01 & 0.01 & 0.01 & 0.01 & .04 & & \\
\hline Género del encuestado & -0.01 & -0.04 & 0.02 & 0.02 & -.02 & & \\
\hline Ingreso mensual hogar & 0.06 & 0.04 & 0.07 & 0.01 & $.21^{* * *}$ & & \\
\hline Nivel de estudios & 0.02 & -0.01 & 0.05 & 0.01 & $.07^{+}$ & & \\
\hline Consumo de juego estratégico & -0.52 & -1.02 & -0.02 & 0.26 & $-.75^{*}$ & & \\
\hline Consumo de asunto político & 0.77 & 0.21 & 1.33 & 0.29 & $1.00^{* *}$ & & \\
\hline
\end{tabular}

Nota: $I C=$ Intervalo de confianza, $L I=$ Límite inferior; $L S=$ Límite superior.

${ }^{*} p<.05 ;{ }^{* *} p<.01 ;{ }^{* * *} p<.001 ;{ }^{+} p<.100$

Al respecto de este primer análisis (tabla 3), las variables utilizadas en la regresión cumplieron el supuesto de independencia respecto de la variable dependiente, en este caso la sofisticación política factual, atendiendo a que el valor del test de Durbin-Watson fue aceptable $(d=1.87)$. Además, la ecuación resultante explicó el $13 \%$ de la varianza de la variable dependiente. Los resultados mostraron un impacto positivo del consumo de noticias con encuadre de asunto político ( $\beta=1.00, p=.007$ ) en el incremento de la sofisticación política factual. Por su parte, el consumo de contenidos informativos donde estaba presente el encuadre de juego estratégico impactó de forma negativa $(\beta=-.75, p=.043)$, es decir, tendió a hacer disminuir el nivel de sofisticación política factual. Por su parte, tanto un mayor nivel de ingresos familiares $(\beta=.21, p<.001)$ como nivel de estudios $(\beta=.07, p=.065)$ contribuyeron a hacer aumentar este tipo de sofisticación política de los ciudadanos.

Tabla 4. Resultados de la regresión jerárquica para la sofisticación política electoral

\begin{tabular}{|c|c|c|c|c|c|c|c|}
\hline & \multirow{2}{*}{ B } & \multicolumn{2}{|c|}{$95 \%$ IC para B } & \multirow{2}{*}{ EE B } & \multirow{2}{*}{$\beta$} & \multirow{2}{*}{$R^{2}$} & \multirow{2}{*}{$\Delta R^{2}$} \\
\hline & & LI & $L S$ & & & & \\
\hline Paso 1 & & & & & & .05 & $.05^{* * *}$ \\
\hline Constante & 2.05 & 1.75 & 2.36 & 0.16 & & & \\
\hline Edad del encuestado & -0.01 & -0.01 & 0.01 & 0.01 & -.03 & & \\
\hline Género del encuestado & -0.04 & -0.11 & 0.02 & 0.03 & -.04 & & \\
\hline Ingreso mensual hogar & 0.09 & 0.05 & 0.13 & 0.02 & $.17^{* * *}$ & & \\
\hline Nivel de estudios & 0.06 & 0.01 & 0.11 & 0.03 & $.09^{*}$ & & \\
\hline Paso 2 & & & & & & .07 & $.02 * * *$ \\
\hline Constante & 1.93 & 1.62 & 2.24 & 0.16 & & & \\
\hline Edad del encuestado & -0.01 & -0.01 & 0.01 & 0.01 & -.01 & & \\
\hline Género del encuestado & -0.03 & -0.09 & 0.04 & 0.03 & -.03 & & \\
\hline Ingreso mensual hogar & 0.09 & 0.05 & 0.13 & 0.02 & $.17^{* * *}$ & & \\
\hline Nivel de estudios & 0.06 & 0.01 & 0.11 & 0.03 & $.08^{*}$ & & \\
\hline Consumo de asunto político & 0.23 & 0.13 & 0.32 & 0.05 & $.15^{* * *}$ & & \\
\hline
\end{tabular}

Nota: $I C=$ Intervalo de confianza, $L I=$ Límite inferior; $L S=$ Límite superior.

${ }^{*} p<.05 ;{ }^{* *} p<.01 ;{ }^{* * *} p<.001$ 
En cuanto al modelo explicativo de la sofisticación política electoral, es decir, el interés y conocimiento de los contenidos y asuntos de corte electoral, el análisis cumplió igualmente con los supuestos de independencia $(d=1.88)$, explicando el modelo únicamente el $7.4 \%$ de la varianza (tabla 4). En este caso, sólo se detectó un impacto significativo y de tipo positivo por parte del consumo del encuadre de asunto político en contenidos informativos durante la campaña ( $\beta=$ $.15, p<.001)$, contribuyendo por tanto al aumento de la sofisticación política electoral. Asimismo, tanto un mayor nivel de ingresos familiares $(\beta=.17, p<.001)$ como nivel de estudios $(\beta=.08, p=.021)$ contribuyeron a hacer aumentar la sofisticación política electoral de los ciudadanos.

Tabla 5. Resultados de la regresión jerárquica para la participación política offline

\begin{tabular}{|c|c|c|c|c|c|c|c|}
\hline & & 95 & ra $B$ & & & & \\
\hline & & $L I$ & $L S$ & 20 & $r$ & 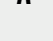 & (11 \\
\hline Paso 1 & & & & & & .04 & $.04 * * *$ \\
\hline Constante & 1.91 & 1.31 & 2.51 & 0.31 & $-.17^{* * *}$ & & \\
\hline Edad del encuestado & -0.01 & -0.02 & -0.01 & 0.01 & $-.12^{* * *}$ & & \\
\hline Género del encuestado & -0.23 & -0.35 & -0.10 & 0.07 & -.02 & & \\
\hline Ingreso mensual hogar & -0.02 & -0.09 & 0.06 & 0.04 & $.09 *$ & & \\
\hline Nivel de estudios & 0.12 & 0.02 & 0.21 & 0.05 & & & \\
\hline Paso 2 & & & & & & .24 & $.20 * * *$ \\
\hline Constante & 1.18 & 0.64 & 1.72 & 0.28 & & & \\
\hline Edad del encuestado & -0.01 & -0.01 & -0.01 & 0.01 & $-.09^{* *}$ & & \\
\hline Género del encuestado & -0.15 & -0.26 & -0.04 & 0.06 & $-.08^{*}$ & & \\
\hline Ingreso mensual hogar & -0.02 & -0.08 & 0.05 & 0.03 & -.02 & & \\
\hline Nivel de estudios & 0.10 & 0.01 & 0.19 & 0.04 & $.08^{*}$ & & \\
\hline Consumo de Asunto Político & 1.35 & 1.18 & 1.53 & 0.09 & $.45^{* * *}$ & & \\
\hline
\end{tabular}

Nota: $I C=$ Intervalo de confianza, $L I=$ Límite inferior; $L S=$ Límite superior.

$* p<.05 ; * * p .01 ; * * *<.001$

Por otra parte, y respecto a los modelos explicativos de la participación política offline (tabla 5), las variables utilizadas en la regresión nuevamente cumplieron el supuesto de independencia respecto de la variable dependiente, atendiendo a que el valor del test de Durbin-Watson fue aceptable $(d=1.95)$. Además, la ecuación resultante explicó hasta un $24 \%$ de la participación política presencial u offline. Los resultados mostraron un impacto positivo del consumo de contenidos con encuadres de asunto político durante las elecciones $(\beta=.45, p<.001)$, haciendo que aumentaran las actividades políticas presenciales de los participantes. Además, también se observó que a menor edad del encuestado $(\beta=-.09, p=$ $.002)$, si el encuestado era hombre $(\beta=-.08, p=.010)$ y si mantenía un mayor nivel de estudios $(\beta=.08, p=.022)$, el nivel de participación política real tendía a aumentar.

Tabla 6. Resultados de la regresión jerárquica para la participación política online

\begin{tabular}{|c|c|c|c|c|c|c|c|}
\hline & \multirow{2}{*}{ B } & \multicolumn{2}{|c|}{$95 \%$ IC para B } & \multirow{2}{*}{ EE B } & \multirow{2}{*}{$\boldsymbol{\beta}$} & \multirow{2}{*}{$R^{2}$} & \multirow{2}{*}{$\Delta R^{2}$} \\
\hline & & $L I$ & LS & & & & \\
\hline Paso 1 & & & & & & .03 & $.03^{* * *}$ \\
\hline Constante & 2.28 & 1.60 & 2.96 & 0.35 & & & \\
\hline Edad del encuestado & -0.01 & -0.01 & 0.01 & 0.01 & $-.10^{* *}$ & & \\
\hline Género del encuestado & -0.26 & -0.41 & -0.12 & 0.07 & $-.12^{* * *}$ & & \\
\hline Ingreso mensual hogar & 0.03 & -0.06 & 0.12 & 0.04 & .03 & & \\
\hline Nivel de estudios & 0.09 & -0.02 & 0.20 & 0.06 & .06 & & \\
\hline Paso 2 & & & & & & .23 & $.20 * * *$ \\
\hline Constante & 1.44 & 0.82 & 2.05 & 0.31 & & & \\
\hline Edad del encuestado & 0.01 & -0.01 & 0.00 & 0.01 & -.02 & & \\
\hline Género del encuestado & -0.17 & -0.30 & -0.05 & 0.07 & $-.08^{* *}$ & & \\
\hline Ingreso mensual hogar & 0.03 & -0.05 & 0.11 & 0.04 & .03 & & \\
\hline Nivel de estudios & 0.07 & -0.03 & 0.17 & 0.05 & .05 & & \\
\hline Consumo de asunto político & 1.55 & 1.36 & 1.75 & 0.10 & $.46^{* * *}$ & & \\
\hline
\end{tabular}

Nota: $I C=$ Intervalo de confianza, $L I=$ Límite inferior; $L S=$ Límite superior.

$* p<.05 ; * * p .01 ; * * * p<.001$

Finalmente, en relación con el modelo explicativo de la participación política online, el análisis cumplió igualmente con los supuestos de independencia $(d=1.88$ ), explicando el modelo el $23.1 \%$ de la varianza (tabla 6$)$. En este sentido, de 
nuevo esta participación aumentó en la medida en que se consumía más el encuadre de asunto político en los contenidos informativos para informarse de la campaña electoral $(\beta=.46, p<.001)$. También tendía a aumentar la participación política online cuando el encuestado era hombre $(\beta=-.08, p=.006)$. Como se puede comprobar en los datos obtenidos en el estudio (tablas 5 y 6 ), en ningún tipo de participación política se observó que influyera el consumo de encuadre de juego estratégico durante la campaña electoral.

\section{Discusión y conclusiones}

En el presente artículo se planteó como objetivo determinar cuál es la influencia del consumo de información con tratamiento ya sea de juego estratégico o de asunto, en el desarrollo del compromiso político de la ciudadanía, tomando como proxy de éste las actitudes y comportamientos como la sofisticación y la participación políticas. Para ello, se trabajó en el contexto de la campaña electoral presidencial mexicana de 2018 , con datos provenientes de un análisis de contenido de la cobertura ofrecida por la televisión y la prensa escrita de esta campaña, así como una encuesta panel realizada en dos momentos del proceso electoral: durante el mes previo a la elección e inmediatamente después a la finalización del proceso y elección del nuevo presidente de México.

Para dar cumplimiento a este objetivo, el trabajo se planteó responder varias preguntas de investigación. Con relación a la primera de las mismas, que cuestiona el impacto de dicha información específicamente en el nivel de sofisticación política factual, los resultados obtenidos permitieron determinar un impacto significativo del consumo de información enfocada con el encuadre de asunto. Sin embargo, el consumo de noticias encuadradas desde el juego estratégico tuvo un impacto negativo sobre esta sofisticación política factual de la ciudadanía. Por su parte, la segunda pregunta de investigación planteada buscaba determinar en qué medida el consumo de información bajo los encuadres mencionados impactó en el nivel de sofisticación política electoral de la ciudadanía. Los resultados mostraron que fue el consumo de asunto político el que llegó a determinar un cambio en este tipo de sofisticación política ciudadana.

Estos resultados suponen un primer acercamiento al estudio de los efectos que el consumo de los encuadres noticiosos tiene sobre la sofisticación política, en particular desde un diseño de realismo experimental. Como ya se indicó anteriormente, estudios como el realizado por Shehata (2014) han demostrado el impacto positivo del consumo del encuadre de asunto político en el interés ciudadano hacia aquello que ocurre en la esfera pública. Sin embargo, no se han detectado estudios que vinculen el consumo de encuadres con el desarrollo de sofisticación política, entendida ésta como una variable dependiente o criterio. Por ello, resulta importante la constatación del impacto favorable en la sofisticación del ciudadano, tanto a nivel factual como electoral, que tiene el consumo de contenidos enfocados desde el asunto político, lo que contribuye al aumento del conocimiento de las propuestas programáticas y debates sobre políticas públicas. Algo que puede redundar en una orientación hacia la acción y el involucramiento políticos (Delli-Carpini, 2004). Por otra parte, el impacto negativo del consumo de juego estratégico se focalizó en la sofisticación factual, lo que podría redundar en efectos negativos indirectos, como el incremento de actitudes políticas cínicas o desafectas (De-Vreese et al., 2011).

En cuanto a la tercera y cuarta preguntas de investigación del estudio, en ambas se planteaba la duda acerca del impacto del consumo de ambos encuadres en la participación política, disociando la misma entre la actividad desarrollada en el mundo offline y en el online. En ambos casos, únicamente el consumo de información encuadrada desde el asunto político tuvo la capacidad de hacer incrementar ambos tipos de participación. Así, y al igual que ocurriera con respecto a la sofisticación, el involucramiento político de los ciudadanos en los terrenos presencial y digital tendió a aumentar durante la campaña electoral en la medida en que consumieron contenidos informativos donde se planteaban propuestas programáticas y se debatía acerca de éstas. El resultado se ajusta, por tanto, a lo planteado en otros contextos acerca del estímulo positivo que este tipo de información puede tener para el compromiso ciudadano (De-Vreese; Semetko, 2002). Sin embargo, el encuadre de juego estratégico no influyó ni positiva ni negativamente en el nivel de participación política mantenida por los ciudadanos durante la campaña.

Los resultados obtenidos muestran un panorama que se acerca más a lo planteado por los postulados de la movilización política que del malestar mediático. En general, el consumo de framing político se presenta como un predictor importante para explicar el desarrollo de compromiso político durante la campaña electoral, sobre todo el consumo de noticias tratadas desde el asunto, que privilegia la presentación de propuestas para resolver problemas reales, sus causas y consecuencias, y las propuestas y el debate acerca de las mismas. Este encuadre tuvo la capacidad de hacer incrementar la sofisticación política, es decir, el compromiso cognitivo del ciudadano con la misma, así como la participación política -o compromiso de acción - tanto en el mundo real como el digital (Yung; Leung, 2014). Estos resultados parecen sugerir que el compromiso político que se está desarrollando y potenciando desde los medios con sus contenidos implica un compromiso de acción, el cual es desarrollado con elementos cognitivos de base. Con todo, es también relevante que 
el encuadre de juego estratégico, criticado reiteradamente por banalizar la política y tener efectos sobre el cinismo (Cappella; Jamieson, 1997; De-Vreese, 2005; De-Vreese; Semetko, 2002, Rhee, 1997), no registre efectos significativos en el presente estudio.

Sin embargo, los resultados de este estudio deben ser tomados con cautela pues éstos derivan de un primer acercamiento al análisis de este efecto framing desde una metodología basada en el realismo experimental en el contexto, no solo mexicano, sino también latinoamericano. Si bien en el ámbito internacional ya son varios los ejercicios realizados tomando esta metodología como base para su diseño (Boomgaarden; Kritzinger, 2017; Geers et al., 2017; Schuck et al., 2016; Shehata, 2014; Wettstein, 2012), no puede decirse lo mismo en el contexto latinoamericano, incluso hispanoamericano, de manera que no disponemos de elementos de contraste en otros trabajos. A pesar de ello, y como se ha demostrado con este artículo, este tipo de estrategias de análisis permiten conseguir una fotografía mucho más fiel del efecto de la dieta mediática de los ciudadanos durante los procesos políticos, como puede ser una campaña electoral, en el desarrollo de sus actitudes y comportamientos políticos.

Así, se puede concluir que el estudio abona al cuerpo de trabajos que apoyan la movilización política en el contexto electoral (Díaz-Jiménez; Muñiz, 2017; Muñiz; Maldonado, 2011; Rojas, 1996; Shehata, 2014), pues el consumo mediático, no sólo no ha tendido a generar una ciudadanía apática, desapegada y desinteresada de la política, sino que más bien ha contribuido a que la ciudadanía se interese e involucre en la esfera pública, por lo menos en los momentos políticos como el sometido a análisis en este estudio.

\section{Referencias}

Aalberg, Toril; Strömbäck, Jesper; De-Vreese, Claes H. (2012). "The framing of politics as strategy and game: A review of concepts, operationalizations and key findings". Journalism, v. 13, pp. 162-178.

https://doi.org/10.1177/1464884911427799

Avery, James (2009). "Videomalaise or virtuous circle? The influence of the news media on political trust". International Journal of Press/Politics, v. 14, n. 4, pp. 410-433.

https://doi.org/10.1177/1940161209336224

Boomgaarden, Hajo; Kritzinger, Sylvia (2017). "Election surveys". In: Matthes, Jörg. The international encyclopedia of communication research methods, pp. 1-9. EE.UU.: Wiley. ISBN: 9781118901762

https://doi.org/10.1002/9781118901731.iecrm0077

Buendía, Jorge; Somuano, Fernanda (2003). “Participación electoral en nuevas democracias: la elección presidencial de 2000 en México". Política y gobierno, v. 10, n. 2, pp. 289-322.

http://www.politicaygobierno.cide.edu/index.php/pyg/article/view/342

Cappella, Joseph; Jamieson, Kathleen (1997). Spiral of cynicism: The press and the public good. Oxford University Press. ISBN: 9780195090642

Dalton, Russell (2006). Citizen politics: public opinion and political parties in advanced industrial democracies. USA: CQ Press. ISBN: 9781452203003

De-Vreese, Claes H. (2005). "The spiral of cynicism reconsidered: the mobilizing function of news". European journal of communication, v. 20, n. 3, pp. 283-301.

https://doi.org/10.1177/0267323105055259

De-Vreese, Claes H. (2012). “New avenues for framing research”. American behavioral scientist, v. 56, n. 3, pp. 365-375. https://doi.org/10.1177/0002764211426331

De-Vreese, Claes H.; Boomgaarden, Hajo; Semetko, Holli (2011). “(In)direct framing effects: The effects of news media framing on public support for Turkish membership in the European Union”. Communication research, v. 38, n. 2, pp. 179-205.

https://doi.org/10.1177/0093650210384934

De-Vreese, Claes H.; Semetko, Holli (2002). "Cynical and engaged: Strategic campaign coverage, public opinion, and mobilization in a referendum". Communication research, v. 29, n. 6, pp. 615-641.

https://doi.org/10.1177/009365002237829

Delli-Carpini, Michael (2004). “Mediating democratic engagement: The impact of communications on citizens' involvement in political and civic life". In: Kaid, Lynda-Lee. Handbook of political communication, pp. 395-434. USA: Lawrence Erlbaum. ISBN: 780805837759

Delli-Carpini, Michael; Keeter, Scott (1996). What Americans know about politics and why it matters. USA: Yale University Press. ISBN: 9780300072754 
Díaz-Jiménez, Oniel; Muñiz, Carlos (2017). "Los efectos de la comunicación política en el compromiso político de los jóvenes en la elección presidencial mexicana de 2012". Revista mexicana de ciencias políticas y sociales, v. 62, n. 229, pp. 181-222.

https://doi.org/10.1016/S0185-1918(17)30008-9

Dimitrova, Daniela; Strömbäck, Jesper (2012). "Election news in Sweden and the United States: A comparative study of sources and media frames". Journalism, v. 13, n. 5, pp. 604-619.

https://doi.org/10.1177/1464884911431546

Druckman, James (2001). "The implications of framing effects for citizen competence”. Political behavior, v. 23, pp. 225256.

https://doi.org/10.1023/A:1015006907312

Elenbaas, Matthijs; De-Vreese, Claes H. (2008). "The effects of strategic news on political. Cynicism and vote choice among young voters". Journal of communication, v. 58, n. 3, pp. 550-567.

https://doi.org/10.1111/j.1460-2466.2008.00399.x

Entman, Robert (1993). "Framing: Toward clarification of a fractured paradigm". Journal of communication, v. 43, n. 4, pp. 51-58.

https://doi.org/10.1111/j.1460-2466.1993.tb01304.x

Geers, Sabine; Bos, Linda; De-Vreese, Claes H. (2017). "Informed switchers? How the impact of election news exposure on vote change depends on political information efficacy". International journal of communication, v. 11, pp. 1857-1878. https://ijoc.org/index.php/ijoc/article/view/6063

Gerth, Matthias; Siegert, Gabriele (2012). "Patterns of consistence and constriction: How news media frame the coverage of direct democratic campaigns". American behavioral scientist, v. 56, n. 3, pp. 279-299.

https://doi.org/10.1177/0002764211426326

Gil de Zúñiga, Homero; Molyneux, Logan; Zheng, Pei (2014). “Social media, political expression, and political participation: Panel analysis of lagged and concurrent relationships". Journal of communication, v. 64, pp. 612-634.

https://doi.org/10.1111/jcom.12103

Holtz-Bacha, Christina (1990). "Videomalaise revisited: Media exposure and political alienation in West Germany". European journal of communication, v. 5, n. 1, pp. 73-85.

https://doi.org/10.1177/0267323190005001005

Matthes, Jörg (2008). "Media frames and public opinion: Exploring the boundaries of framing effects in a two-wave panel study". Studies in communication sciences, v. 8, n. 2, pp. 101-128.

https://doi.org/10.5167/uzh-14296

Matthes, Jörg (2012). "Framing politics: An integrative approach". American behavioral scientist, v. 56, n. 3, pp. $247-259$. https://doi.org/10.1177/0002764211426324

Muñiz, Carlos (2015). "La política como debate temático o estratégico. Framing de la campaña electoral mexicana de 2012 en la prensa digital". Comunicación y sociedad, n. 23, pp. 67-95.

https://doi.org/10.32870/cys.v0i23.64

Muñiz, Carlos; Echeverría, Martín; Rodríguez-Estrada, Alejandra; Díaz-Jiménez, Oniel (2018). “Los hábitos comunicativos y su influencia en la sofisticación política ciudadana". Convergencia, n. 77, pp. 99-123.

https://doi.org/10.29101/crcs.v25i77.9298

Muñiz, Carlos; Maldonado, Lauro (2011). “Entre la movilización y el malestar mediático. Impacto de las prácticas comunicativas en las actitudes políticas de los jóvenes". Perspectivas de la comunicación, v. 4, n. 2, pp. 32-54.

http://publicacionescienciassociales.ufro.cl/index.php/perspectivas/article/view/117

Muñiz, Carlos; Saldierna, Alma; Marañón, Felipe-de-Jesús (2018). “Framing of electoral processes: The stages of the campaign as a moderator of the presence of political frames in the news". Palabra clave, v. 21, n. 3, pp. 740-771.

https://doi.org/10.5294/pacla.2018.21.3.5

Norris, Pipa (2002). "Tuned out voters? Media impact on campaign learning". In: Harvard University-John F. Kennedy School of Government.

https://sites.hks.harvard.edu/fs/pnorris/Acrobat/Tuned\%200ut.pdf

Putnam, Robert (1993). "What makes democracy work?”. National civic review, v. 82, n. 2, pp. 101-107.

https://doi.org/10.1002/ncr.4100820204

Rhee, June (1997). "Strategy and issue frames in election campaign coverage: a social cognitive account of framing effects". Journal of communication, v. 47, n. 3, pp. 26-48.

https://doi.org/10.1111/j.1460-2466.1997.tb02715.x 
Rinke, Eike; Wessler, Hartmut; Löb, Charlotte; Weinmann, Carina (2013). "Deliberative qualities of generic news frames: Assessing the democratic value of strategic game and contestation framing in election campaign coverage". Political communication, v. 30, n. 3, pp. 474-494.

https://doi.org/10.1080/10584609.2012.737432

Rojas, Hernando (2006). “Comunicación, participación y democracia”. Universitas humanística, n. 62, pp. $109-142$. https://revistas.javeriana.edu.co/index.php/univhumanistica/article/view/2208

Rubin, Allen (2010). Statistics for evidence-based practice and evaluation (2 ${ }^{\text {nd }}$ ed.). USA: Brooks/Cole. ISBN: 9780 495602897

Scheufele, Bertram (2006). "Frames, schemata, and news reporting". Communications: The European journal of communication research, v. 31, pp. 65-83.

https://doi.org/10.1515/COMMUN.2006.005

Schuck, Andreas; Boomgaarden, Hajo; De-Vreese, Claes H. (2013). "Cynics all around? The impact of election news on political cynicism in comparative perspective". Journal of communication, v. 63, n. 2, pp. 287-311.

https://doi.org/10.1111/jcom.12023

Schuck, Andreas; Vliegenthart, Rens; De-Vreese, Claes H. (2016). "Who's afraid of conflict? The mobilizing effect of conflict framing in campaign news". British journal of political science, v. 46, n. 1, pp. 177-194.

https://doi.org/10.1017/S0007123413000525

Shehata, Adam (2014). "Game frames, issue frames, and mobilization: Disentangling the effects of frame exposure and motivated news attention on political cynicism and engagement". International journal of public opinion research, v. 26, n. 2, pp. 157-177.

https://doi.org/10.1093/ijpor/edt034

Valentino, Nicholas; Beckmann, Matthew; Buhr, Thomas (2001). "A spiral of cynicism for some: The contingent effects of campaign news frames on participation and confidence in government". Political communication, v. 18, n. 4, pp. 34767.

https://doi.org/10.1080/10584600152647083

Valenzuela, Sebastián; Park, Namsu; Kee, Kerk (2009). "Is there social capital in a social network site?: Facebook use and college students' life satisfaction, trust, and participation". Journal of computer-mediated communication, v. 14, n. 4, pp. 875-901.

https://doi.org/10.1111/j.1083-6101.2009.01474.x

Wettstein, Martin (2012). "Frame adoption in referendum campaigns: The effect of news coverage on the public salience of issue interpretations". American behavioral scientist, v. 56, n. 3, pp. 318-333.

https://doi.org/10.1177/0002764211426328

Yung, Betty; Leung, Lisa (2014). "Facebook as change? Political engagement in semi-democratic Hong Kong in its transition to universal suffrage". Journal of Asian public policy, v. 7, n. 3, pp. 291-305.

https://doi.org/10.1080/17516234.2014.922147

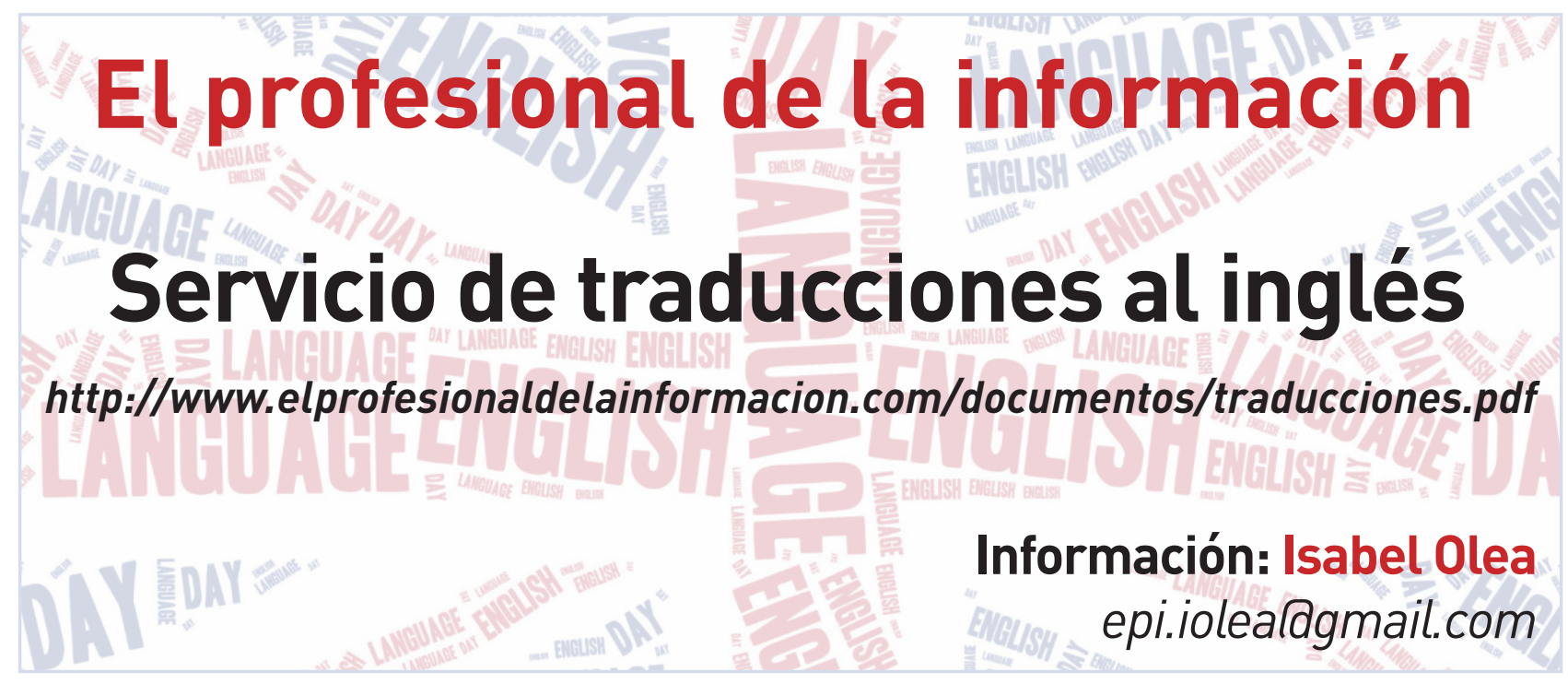

